\title{
Carvedilol in glioma treatment alone and with imatinib in vitro
}

\author{
MINE ERGUVEN $^{1 *}$, NURAY YAZIHAN ${ }^{5}$, ESIN AKTAS $^{2}$, AKIN SABANCI $^{3}$, \\ CHIANG J. LI ${ }^{7}$, GULPERI OKTEM ${ }^{6}$ and AYHAN BILIR ${ }^{4 *}$
}

\begin{abstract}
${ }^{1}$ Department of Biochemistry, Istanbul University, Istanbul Faculty of Medicine, Istanbul 34093, and Faculty of Pharmacy, Istanbul 34116; ${ }^{2}$ Department of Immunology, Istanbul University Institute of Experimental Medicine (DETAE); Departments of ${ }^{3}$ Neurosurgery, and ${ }^{4}$ Histology and Embryology, Istanbul University, Istanbul Faculty of Medicine, Istanbul 34093; ${ }^{5}$ Department of Physiopathology, Ankara University, Faculty of Medicine, Ankara 06100; ${ }^{6}$ Department of Histology and Embryology, Ege University, Faculty of Medicine, Izmir, Turkey;

${ }^{7}$ Division of Gastroenterology, Beth Israel Deaconess Medical Center, Harvard Medical School, Boston, MA 02215, USA
\end{abstract}

Received October 9, 2009; Accepted November 20, 2009

DOI: 10.3892/ijo_00000563

\begin{abstract}
The purpose of the study was to investigate whether carvedilol has an antiproliferative effect alone and whether carvedilol provides an additive, synergistic or antagonistic effect on imatinib mesylate-induced cytotoxicity in both C6 glioma monolayer and spheroid culture. The C6 rat glioma chemoresistant experimental brain tumour cell line, that is notoriously difficult to treat with combination chemotherapy, was used both in monolayer and spheroid cultures. We treated C6 glioma cells with carvedilol alone and a combination of carvedilol and imatinib mesylate at a concentration of $10 \mu \mathrm{M}$. Following treatment, we evaluated cell proliferation index, bromodeoxyuridine labelling index (BrDU-LI), cell cycle distributions, apoptotic cell percentages, cAMP levels and three dimensional cell morphology at monolayer cultures. In addition BrDU-LI, volume and morphology of spheroids were also assessed. Carvedilol and imatinib mesylate alone reduced cell number, BrDU-LI, cAMP levels and spheroid volume. Carvedilol and imatinib mesylate arrested cells at G0/ G1 phase in a time-dependent manner and time-independent manner, respectively. Carvedilol increased apoptosis rate only at the 24th h, but imatinib mesylate did for all time intervals. Interestingly carvedilol, drug with well-known protective effect on mitochondria, induced severe mitochondria damage, and imatinib mesylate induced autophagy confirmed only by transmission electron microscopy. These
\end{abstract}

Correspondence to: Dr Mine Erguven, Department of Biochemistry, Istanbul University, Istanbul Faculty of Medicine, Istanbul 34093, and Faculty of Pharmacy, Istanbul 34116, Turkey E-mail: mine.erguven@gmail.com; merguven@istanbul.edu.tr

${ }^{*}$ Contributed equally

Key words: carvedilol, imatinib mesylate, C6 glioma, mitochondria damage, autophagy, spheroid results suggest that carvedilol showed antitumour activity against rat C6 glioma cells and a combination of carvedilol with imatinib mesylate resulted in enhanced in vitro antitumour activity.

\section{Introduction}

Receptor and non-receptor tyrosine kinases (TKs) have emerged as clinically useful target molecules for therapeutic cancer treatment (1). Imatinib mesylate (IM; STI-571, Gleevec $^{\circledR}$ ) an ATP analogue, potently and selectively inhibits several protein tyrosine kinase receptors for platelet-derived growth factor (PDGF), including breakpoint cluster regionabelson (Bcr-Abl) and c-kit by interacting with their ATPbinding site $(2,3)$. Mitochondria and telomerase are also targets of IM $(4,5)$. IM was primarily designed to treat chronic myelogenous leukemia (CML), and is currently the drug of choice for metastatic gastrointestinal stromal tumors $(6,7)$. The success of this compound in treating CML has led to broader studies of its application in treating other tumors, such as glioblastoma (GBM), small lung cancer, anaplastic thyroid cancer and prostate cancer (8-11).

C6 rat glioma is a chemoresistant experimental brain tumour cell line (12). This cell line has been widely used to study human GBM as the two cancers have many similar properties (13). IM has recently been shown to be efficacious in preclinical studies for GBM. Unfortunately, the potential utility of IM has been hampered by problems with drug distribution to the nervous system, as IM is a substrate of P-glycoprotein ( $\mathrm{p}-\mathrm{Gp}$; the product of MDR1 gene), an active efflux transporter, thus limiting the distribution of IM to the central nervous system (14). Fortunately, the use of p-Gp inhibitors to improve brain penetration of $\mathrm{p}-\mathrm{Gp}$ substrate drugs has been shown to be effective in both animal and human studies (15).

Carvedilol (CAR; Dilatrend ${ }^{\circledR}$ ) is a cardiovascular drug licensed for the treatment of chronic heart failure. CAR has multifaceted therapeutic potential including anti-oxidant and anti-neoplastic action. CAR has been shown to inhibit the 
cellular proliferation of various human cell lines in vitro, including myelogenous leukemia K562, cervix carcinoma HeLa, melanoma Fem-X, breast tumour and osteosarcoma MG63. Additionally, CAR is able to reverse multidrug resistance to anticancer drugs through $\mathrm{p}-\mathrm{Gp}$ inhibition. The major molecular targets of CAR include the membrane adrenoreceptors $(\beta-1, \beta-2$ and $\alpha-1)$, growth factor receptors including platelet-derived growth factor receptors (PDGFr), reactive oxygen species, ion channels $\left(\mathrm{K}^{+}\right.$and $\left.\mathrm{Ca}^{2+}\right)$, p-Gp and mitochondria (16-22).

Previous studies have shown that compared to cancer cells cultured under conventional two-dimensional (2D) monolayer conditions, cancer cells grown in vitro as threedimensional (3D) spheroids, more accurately mimic the drug sensitivity and/or the drug resistance behaviours of solid tumors in vivo (23). In the present study we investigated whether carvedilol has an antiproliferative effect alone and whether CAR provides an additive, synergistic or antagonistic effect on IM-induced cytotoxicity in both C6 glioma 2D monolayer and 3D spheroid cultures.

\section{Materials and methods}

Drugs and solutions. The IM was kindly provided by Dr E. Buchdunger (Novartis AG, Basel, Switzerland) and Dr Barbaros Durgun (Novartis AG, Istanbul, Turkey) and CAR was obtained from Boehringer Mannheim GmbH. These agents were prepared as $200 \mu \mathrm{M}$ stock solutions in doubledistilled water.

Cell culture. The C6 rat glioma cell line was supplied by the American Type Culture Collection (ATCC; Rockville, USA) and was grown in monolayer culture in Dulbecco's modified Eagle's medium-F12 (DMEM-F12; Biological Industries, Israel) supplemented with $10 \%$ heat-inactivated foetal calf serum, $100 \mathrm{U} / \mathrm{ml}$ penicillin and $100 \mu \mathrm{g} / \mathrm{ml}$ streptomycin (Sigma Chemical Co., St Louis, MO). Cells in semi-confluent flasks were harvested using $0.05 \%$ trypsin (Sigma Chemical Co.) and centrifuged after the addition of DMEM-F12 for trypsin inactivation and then resuspended in culture medium. Following trypan blue exclusion assay, glioma cells were plated in six-well culture plates containing $5 \mathrm{ml}$ DMEM-F12 medium at a concentration of $5 \times 10^{5}$ cells/well with $100 \%$ vitality.

Constitution of a 3-dimensional spheroid model. An in vitro multicellular C6 glioma spheroid model was established using a liquid overlay technique. Briefly, semi-confluent monolayer cell cultures were trypsinized and single cells with $100 \%$ vitality were cultured over $3 \%$ noble agar-coated (Difco, USA) six-well culture plates containing $5 \mathrm{ml}$ DMEMF12 medium at a concentration of $1 \times 10^{6}$ cells/well (24).

Experimental design. The two drugs under investigation were applied at a volume of $100 \mu \mathrm{l}$ to monolayer and spheroid cultures of malignant C6 glioma cells in concentrations ranging from 1 to $200 \mu \mathrm{M}$, while the negative control cells received nutrient medium alone. Cultures were incubated for 24, 48, 72 and $96 \mathrm{~h}$. The concentration of drugs effective on the cancer cell line was determined as $10 \mu \mathrm{M}$. Experiments were performed in both monolayer and spheroid cultures as follows: 1) untreated control cells, 2) cells treated with IM, 3 ) cells treated with CAR, 4) cells treated with a combination of IM and CAR. The effects of the drugs on the monolayer cell cultures were assessed by evaluating cell proliferation, cell morphology using scanning electron microscopy (SEM), cyclic adenosine monophosphate (cAMP) levels, cell death analysis, cell cycle kinetics by flow cytometry [Annexin-Vfluorescein isothiocyanate/propidium iodide (Annexin-VFITC/PI) staining] and immunohistochemistry [bromodeoxyuridine labelling index (BrDU-LI) values] while the effects of the drugs on the spheroid cultures were determined by evaluating spheroid volumes and spheroid morphology using transmission electron microscopy (TEM).

Spheroid growth measurement. Spheroid growth was monitored by measuring the increase in spheroid size over $24 \mathrm{~h}$. The size of nearly 20 spheroids was evaluated in each group by measuring two orthogonal diameters ( $a$ and $b$ ) using line morphometry function. The measurements were performed by two independent readers, blinded to drug-treatment design. The volume was calculated in $\mu \mathrm{m}^{3}$ using the formula $\mathrm{V}=4 / 3[(\mathrm{a} / 2+\mathrm{b} / 2) / 2]^{3}$, where $\mathrm{a}$ is the maximum diameter and $\mathrm{b}$ is the minimum diameter (25).

\section{Cell cycle kinetics}

DNA synthesis (S phase) analyses of BrdU labelling index in monolayer culture (immunohistochemistry). C6 rat glioma cells were seeded on glass cover slips and treated with drugs, as described in the survival studies, every $24 \mathrm{~h}$ for a total of $96 \mathrm{~h}$. Endogenous peroxidase activity was quenched with $0.5 \% \mathrm{H}_{2} \mathrm{O}_{2}$ in methanol. Double-stranded DNA was then denatured with $4 \mathrm{~N}$ hydrochloric acid $(\mathrm{HCl})$. Following washings, a non-specific blocking reagent (Ultra-V-Block Lab Vision Co., Westinghouse, CA) was used to prevent non-specific binding. Monoclonal mouse anti-bromodeoxyuridine (BrdU) antibody (Lab Vision Co., 1:50) was the primary antibody, and secondary antibody was biotinylated goat anti-mouse (Lab Vision Co.). After washing, peroxidaseconjugated streptavidin (Lab Vision Co.) was applied and aminoethyl carbazole (AEC) was used as the chromogen. The slides were then counterstained with Mayer's hematoxylin to enhance nuclear staining. BrDU labelled cells in $\mathrm{S}$ phase showed red-stained nuclei. Negative control samples were processed in a similar manner but excluding the primary antibodies. BrdU labeling index (the number of positivelystained cells divided by the total number of cells) was calculated by evaluating at least 3000 cells in multiple highpower fields.

DNA synthesis (S phase) analysis of BrdU labelling index in spheroid cultures (immunohistochemistry). Spheroids were gently removed from the surface of the solidified agarose and then treated with a graded ethanol series $(70,80,90,96$ and $100 \%)$ and toluol, then embedded in paraffin. Sections were cut by the microtome (Leica MR 2145, Heerbrugg, Switzerland) and were attached to a slide coated with poly-1lysine. Sections were treated with $0.1 \%$ trypsin for antigen retrieval and incubated in a solution of $0.3 \% \mathrm{H}_{2} \mathrm{O}_{2}$. Then they were treated with $4 \mathrm{~N} \mathrm{HCl}$ and incubated in $0.1 \mathrm{M}$ Borax. 
Following washings, a non-specific blocking reagent (UltraV-Block, Lab Vision Co.) was used to prevent non-specific binding. Monoclonal mouse anti-BrdU antibody (Lab Vision Co., 1:50) was used as primary antibody, and biotinylated goat anti-mouse (Lab Vision Co.) as the secondary antibody. Following washing, peroxidase-conjugated streptavidin (Lab Vision Co.) was applied and aminoethyl carbazole was used as the chromogen. Sections were counterstained with Mayer's hematoxylin. BrDU labelled cells in S phase had red-stained nuclei. Approximately 30 spheroids in each slide were selected for calculation of the BrDU labelling index. For negative controls, adjacent sections were processed excluding the primary antibody.

Cell cycle analysis (flow cytometry). The effects of drugs on the cell cycle were examined using a DNA analysis kit (BD Pharmingen, San Diego, CA, USA) according to the manufacturer's instructions. Briefly, C6 glioma cells were induced at a cell density of $5 \times 10^{5}$ cells $/ \mathrm{ml}$ in the presence of each drug applied separately and in combination for different time intervals ( 24 and $72 \mathrm{~h}$ ). Cells were then harvested, centrifuged, washed and resuspended in buffer [dimethyl-sulfoxide (DMSO) in sucrose-sodium citrate] for $5 \mathrm{~min}$ at room temperature. A mixture of trypsin in spermine tetrahydrochloride detergent buffer was added and samples were incubated for 20 min at room temperature. After the addition of RNase A and trypsin inhibitor in spermine buffer cells were incubation with propidium iodide, in the dark, for $20 \mathrm{~min}$ at $4^{\circ} \mathrm{C}$. Finally, flow cytometric analysis was performed immediately using a FACScan flow cytometer (FACS Diva, Beckton-Dickinson, CA, USA) and fluorescence intensity data were acquired using the instruments' operating software (CellQuest; BD Pharmingen). The percentages of the analyzed cell population in G0/G1-, S-, or G2/M-phases was determined by the Mod Fit cell cycle analysis program.

Assessment of cAMP levels. Following centrifugation, the supernatant was removed and $0.1 \mathrm{~N} \mathrm{HCl}$ with DMEM-F12 medium (1:1) was added to cells to stop the reaction at each 24-h interval. Briefly, $25 \mu \mathrm{l}$ of the samples were used to measure cAMP levels. cAMP accumulation was measured in the supernatants according to the method previously described, but with some modifications (26). cAMP was determined by radio-immunoassay using the acetylation protocol. Highaffinity rabbit anti-cAMP antibodies were raised in our laboratory by using BSA-conjugated cAMP. Succinyl-cAMP tyrosine methylester (ScAMP-TME) was iodinated by the chloramine-T method. Mono- and di-iodo ScAMP-TME were used as tracer ligands for the radioimmunoassay (RIA), and were then purified by gel-filtration chromatography (sephadex G 25 superfine), equilibrated and eluted with $1 \mathrm{M}$ sodium acetate $(\mathrm{pH} 5)$. The results were given in $\mathrm{pmol} / \mathrm{ml}$.

Apoptotic cell death analysis (flow cytometry). One of the manifestations of apoptosis is the translocation of phosphotidylserine (PS) from the inner membrane to the outer side of the plasma membrane. Externalization of PS was studied by the Annexin V-binding assay. Briefly, cells were washed twice with PBS and resuspended by binding buffer containing $0.01 \mathrm{M}$ HEPES, $0.14 \mathrm{mM} \mathrm{NaCl}$ and $2.5 \mathrm{Mm} \mathrm{CaCl}_{2}$. A cell suspension $\left(1 \times 10^{5}\right.$ cells in $\left.100 \mu \mathrm{l}\right)$ in binding buffer was incubated with $5 \mu$ of FITC-labeled Annexin V (BD Pharmingen) dye, and propidium iodide for $15 \mathrm{~min}$ in the dark at room temperature. After incubation, the propidium iodide (PI) fluorescence and Annexin V were measured simultaneously in a BD FACSCalibur and analyzed with the instruments' operating software (CellQuest; BD Pharmingen). Data acquisition and analysis were undertaken with CellQuest and WinMDI programs.

Transmission electron microscopy. Harvested spheroids were fixed with $2.5 \%$ glutaraldehyde in $0.1 \mathrm{M}$ sodium cacodylate buffer and post-fixed in $1 \%$ osmium tetraoxide in $0.1 \mathrm{M}$ sodium cacodylate buffer for $1 \mathrm{~h}$ at $4^{\circ} \mathrm{C}$. Cells were incubated in $1 \%$ uranyl acetate for $1 \mathrm{~h}$ at $4^{\circ} \mathrm{C}$, dehydrated in a graded acetone series and embedded in Epon 812. Samples were cut using a rotating blade microtome (Leica) and $70 \mathrm{~nm}$-thick sections were mounted on copper grids. Sections were subsequently stained with 5\% uranyl acetate and counterstained with Reynold's lead citrate. Sections were examined with a Jeol-Jem 1011 transmission electron microscope.

Scanning electron microscopy. C6 glioma cells were seeded on microslides in 24-well culture plates containing $1 \mathrm{ml}$ of DMEM-F12 medium at a concentration of $5 \times 10^{4}$ cells/well. After cell attachement, drugs at a concentration of $10 \mu \mathrm{M}$ were applied to the cell cultures individually and in combination. Cells were fixed with $2.5 \%$ glutaraldehyde in $0.1 \mathrm{M}$ of sodium cacodylate buffer ( $\mathrm{pH} 7.4$ ) for $1 \mathrm{~h}$ at $4^{\circ} \mathrm{C}$ at $24 \mathrm{~h}$ and $72 \mathrm{~h}$. Cells were washed twice for $10 \mathrm{~min}$ with $0.1 \mathrm{M}$ of sodium cacodylate buffer and post-fixed in $1 \%$ osmium tetraoxide for $1 \mathrm{~h}$ at $4^{\circ} \mathrm{C}$. The cells were dehydrated in a graded acetone series and incubated in amyl acetate. Microslides were critical-point dried, sputter-coated with gold-palladium and observed by scanning electron microscopy (Jeol-JSM-5200). Photographs were taken at $25 \mathrm{kV}$.

Statistical analysis. All the results, with the exception of cAMP levels, were statistically analyzed using the Student's t-test. Alterations in cAMP levels were assessed by One way Anova test with post hoc analysis using Tukey HSD at $24 \mathrm{~h}$ and Welch Test with Dunnett T3 test at 72 h. Data are presented as mean \pm standard error of mean (SEM) or standard deviation (SD), as appropriate, and at least in triplicate. Results were considered significant with $\mathrm{p}<0.05$.

\section{Results}

Combination treatment of imanitib mesylate and carvedilol results in synergistic inhibition of C6 glioma cell growth. We observed the untreated control group cell culture growing one log point throughout the $72 \mathrm{~h}$ of culture. The drug treatment reduced cell count both individually and in combination. The effects of IM and CAR treatment, when individually administered, resulted in appreciable cell growth inhibition by $72 \mathrm{~h}$, with IM alone inhibiting cell growth significantly at 24 and $72 \mathrm{~h}(\mathrm{p}<0.01)$. When applied separately, CAR reduced cell numbers significantly and in a similar manner to IM after $24(\mathrm{p}<0.05)$ and $72 \mathrm{~h}(\mathrm{p}<0.01)$. In contrast, the reduction in the number of cells following the single applica- 

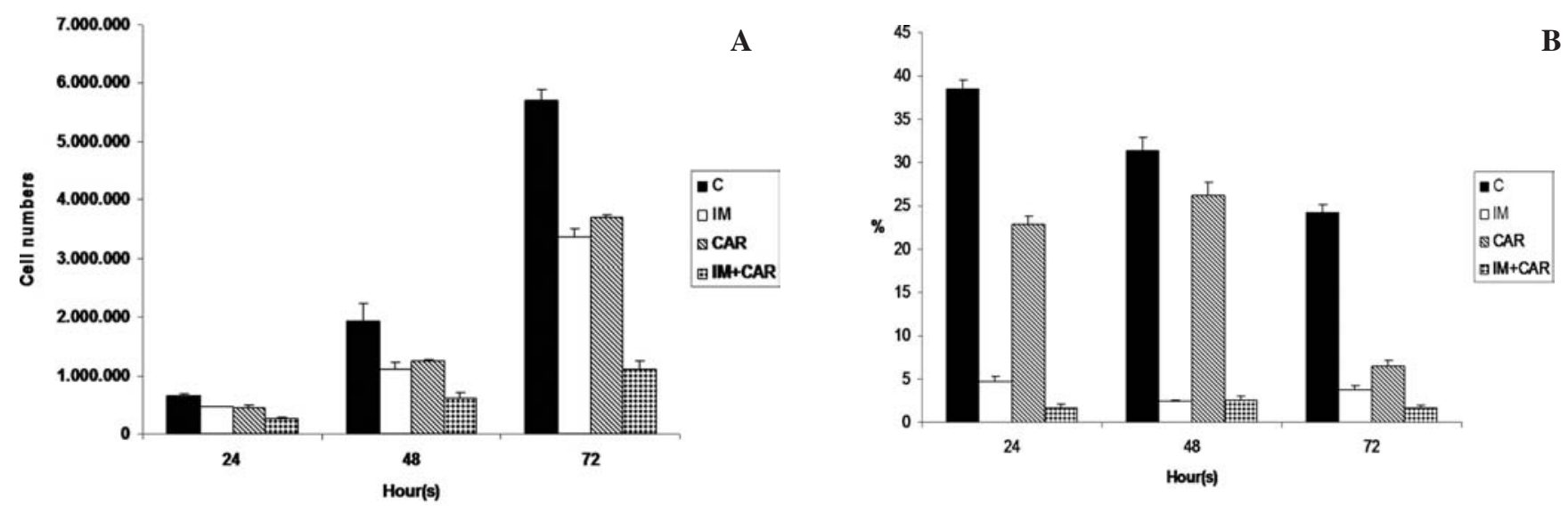

Figure 1. Growth and BrDU-LI results of the monolayer culture C6 glioma cells. (A) Cell growth assay, (B) BrDU-LI assay. Cells were initially plated at a seeding density of $5 \times 10^{5}$ cells per well. C, control; IM, imatinib mesylate; CAR, carvedilol; IM+CAR, imatinib mesylate with carvedilol. Results are mean of three different experiments and presented as mean \pm standard error mean (SEM).

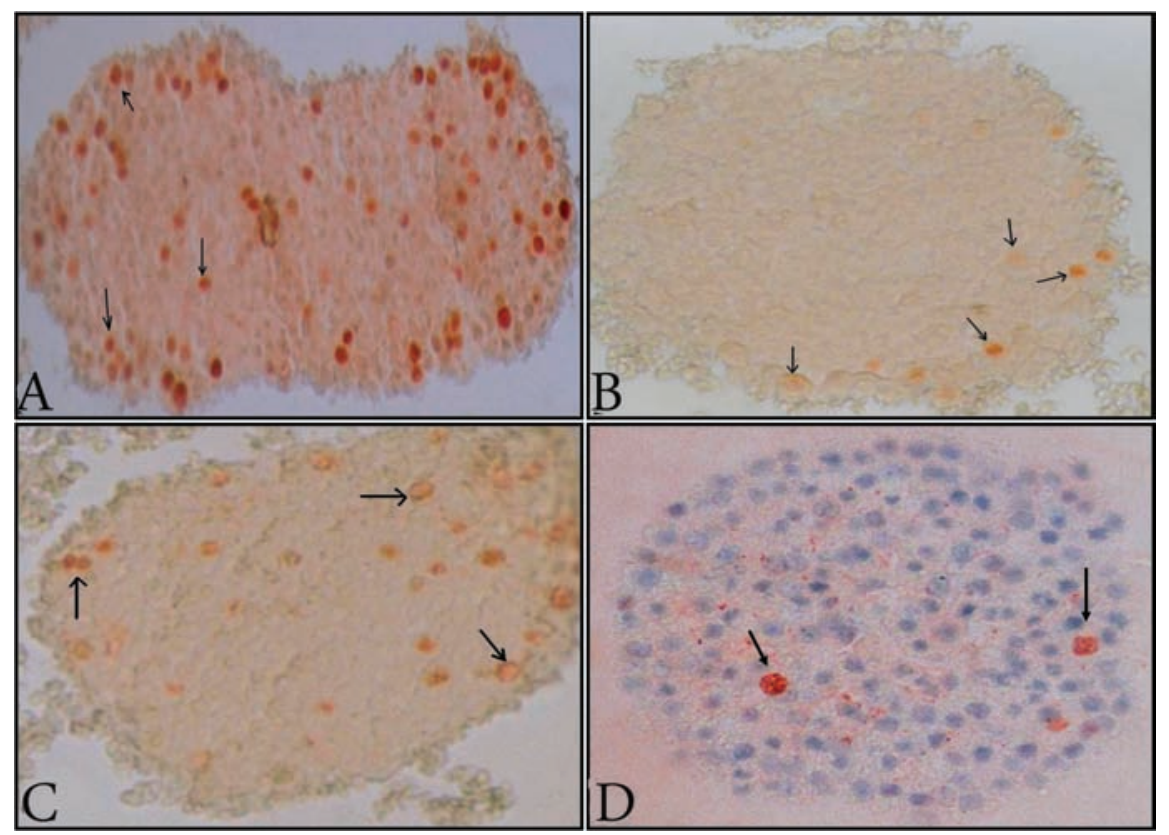

Figure 2. Light microscopy photographs of BrDU labelled spheroids at 24 hours. Cells in spheroids with red-stained nucleus were considered BrDU positive. The arrows show BrDU positive cells. (A) The control group (original magnification, $x 40$ ). (B) The imatinib mesylate group (original magnification, $x 40$ ). (C) The carvedilol group (original magnification, x25). (D) The combination group (original magnification, x25).

tion of drugs and combination application were different. Treatment with IM in combination with CAR resulted in a significant potent reduction in the number of cells at $24 \mathrm{~h}$ $(\mathrm{p}<0.001)$ and at $72 \mathrm{~h}(\mathrm{p}<0.001)$ which was $\sim 6$-fold greater than individual drug treatment, showing synergistic results (Fig. 1A).

Imanitib mesylate and carvedilol combination treatment reduces BrDU-LI in C6 glioma cell monolayer culture. BrDU staining was used to assess DNA synthesis in C6 glioma cell cultures. Cells with red-stained nuclei represent BrDU DNA uptake and thus give positive staining. BrDUpositive staining of the untreated control group glioma cells reduced over time in culture for $72 \mathrm{~h}$, presumably due to the growing population of cells exhausting the same nutritional pool. IM treatment significantly reduced BrDU-LI $(\mathrm{p}<0.001)$ throughout the $72 \mathrm{~h}$ of treatment. In the first $24 \mathrm{~h}$ of treatment, CAR significantly reduced BrDU uptake and at $72 \mathrm{~h}$ of treatment. CAR induced a significant sharp decrease of BrDU-LI $(p<0.001)$ achieving nearly the same effect as IM treatment with a reduction of BrDU-LI by at least 5-fold that of the control. IM combined with CAR significantly inhibited BrDU-LI accumulation resulting in a 20 -fold reduction in BrDU uptake at 24 and $72 \mathrm{~h}(\mathrm{p}<0.001)$ (Fig. 1B).

Imanitib mesylate and carvedilol combination treatment reduces BrDU-LI in C6 glioma cell spheroid culture. Twentyfive spheroids from all experimental groups including the control group were evaluated at $24 \mathrm{~h}$ of culture with BrDU staining. Fig. 2 shows photographs that best represent the 


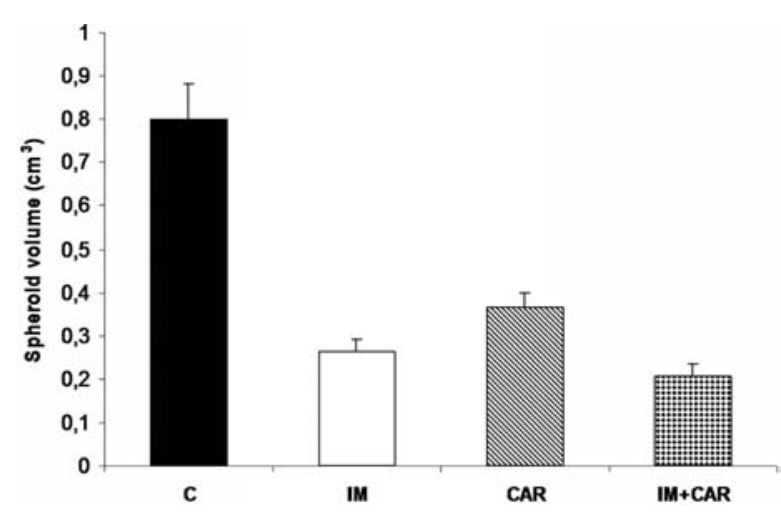

Figure 3. The alterations of spheroid volumes. Cells were initially plated at a seeding density of $1 \times 10^{6}$ cells per well. C, control; IM, imatinib mesylate; CAR, carvedilol; IM+CAR, imatinib mesylate with carvedilol. Results are mean of 20 spheroids and mean of three different experiments. Results are presented as mean $\pm \mathrm{SEM}$ in $\mu \mathrm{m}^{3}$.

obtained results. IM treatment significantly reduced BrDU-LI $(\mathrm{p}<0.001)$ and CAR treatment resulted in a slightly smaller reduction $(\mathrm{p}<0.05)$. When IM was combined with CAR, BrDU-LI reduction was significantly enhanced, providing further evidence for synergistic inhibition of C6 glioma cells by this drug combination $(\mathrm{p}<0.01)$.

Imanitib mesylate and carvedilol combination treatment reduces spheroid volume of C6 glioma spheroids. At $24 \mathrm{~h}$ of culture, spheroids were measured for changes in volume as another means of assessing sensitivity of C6 glioma cells to drug treatment. The volume of spheroids following $24 \mathrm{~h}$ of treatment with IM, CAR, and their combination decreased to $32.67,45$ and $26 \%$, respectively, compared to the untreated control group which was accepted as $100 \%$. All of these reductions were statistically significant $(\mathrm{p}<0.0001)$ (Fig. 3).

Imanitib mesylate and carvedilol combination treatment results in reduced C6 glioma cell metabolic activity as assessed by cAMP levels measurement. cAMP level was measured to assess the metabolic activity of the cells and thus their growth and survival. The cAMP level of the untreated control group culture increased from culture time 0 to $72 \mathrm{~h}$ of culture $(\mathrm{p}<0.0001)$. IM treatment did not inhibit cAMP levels by $24 \mathrm{~h}$ of culture, with cAMP levels being essentially unchanged from those of the control group. However, at $72 \mathrm{~h}$ of treatment, IM treatment inhibited cAMP levels to $50 \%$, which was statistically significant $(\mathrm{p}<0.05)$. CAR treatment resulted in significant reduction of cAMP levels at $24(\mathrm{p}<0.05)$ and at $72 \mathrm{~h}(\mathrm{p}<0.01)$. The combination of IM and CAR reduced cAMP levels at $24 \mathrm{~h}(\mathrm{p}>0.05)$. The most potent inhibitory effect on cAMP was observed in combination treatment group at $72 \mathrm{~h}$, where combination treatment potently and significantly reduced cAMP levels $(\mathrm{p}<0.01)$. These results support the notion that combination treatment of IM and CAR synergistically reduces metabolic cellular activity (Fig. 4).

Imanitib mesylate and carvedilol combination treatment induces apoptosis in C6 glioma cells. The percentage of apoptotic cells in the IM-treated group was moderately increased (7.19 and 5\%) when compared with the control

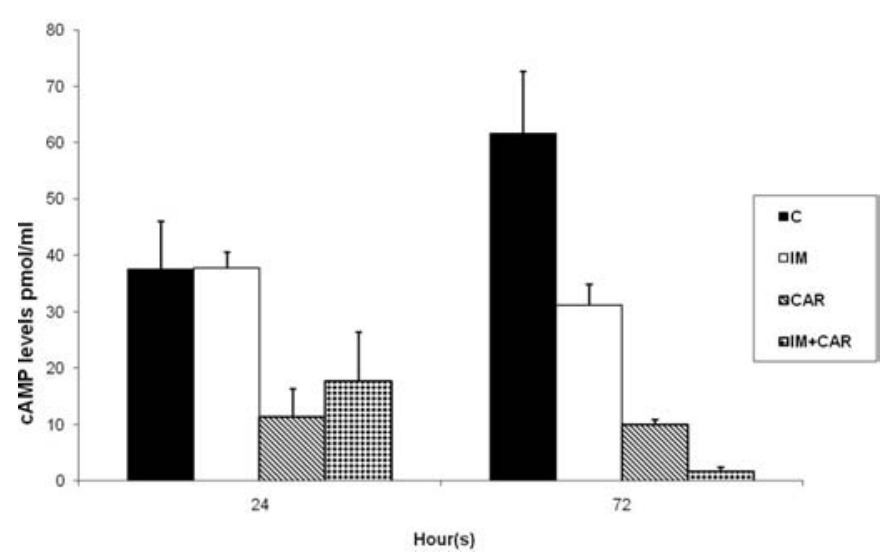

Figure 4. The effects of drugs on cAMP levels of monolayer cultured C6 glioma cells. C, control; IM, imatinib mesylate; CAR, carvedilol; IM+CAR, imatinib mesylate with carvedilol. The results are given in $\mathrm{pmol} / \mathrm{ml}$.

group $(3 \%)$ at 24 and $72 \mathrm{~h}$ of treatment, respectively. Compared to the control group, the percentage of apoptotic cells in the CAR-treated group (5\%) was also mildly increased following $24 \mathrm{~h}$ of treatment, but by $72 \mathrm{~h}$ of treatment, the CAR effects lessened to $2 \%$ of apoptotic cells. When IM and CAR were used in combination, apoptosis was enhanced with the effects appearing greater than can be accounted for by their additive $(21 \%)$ effects alone, assessed by the proportion of apoptotic cells in the untreated control group and individual drug treatment groups following $24 \mathrm{~h}$ of treatment. The percentage of apoptotic cells induced by IM/CAR combination treatment declined by $72 \mathrm{~h}$ of treatment. Potentiation of IM-induced apoptosis by CAR in the combination group treatment could be due to the faciliation of IM penetration through $\mathrm{P}-\mathrm{gp}$ inhibition, activation of the apoptosis cascade through mitochondrial damage and an increase of lipid peroxidative damage of the plasma membrane. The decrease of the combination group efficiency at $72 \mathrm{~h}$ of treatment may be due to a short half-life of CAR $(8 \mathrm{~h})$. Concomitant with other findings of cell growth, BrdU-LI and cAMP levels, these results strengthen our hypothesis of CAR synergism with IM (Fig. 5A).

Imanitib mesylate and carvedilol combination treatment induces autophagy as well as alters cellular morphology of C6 glioma cells in monolayer and spheroid cultures. We expected that differences in cell growth and metabolic state of C6 glioma cells with IM and CAR treatment would be accompanied by morphological changes. Thus, we examined ultrastructural differences between the control and drugtreated groups using TEM and SEM. Our findings indicate that IM and CAR combination may induce autophagic cell death [programmed cell death (PCD) type II] in C6 glioma cells. Autophagy is a process in which subcellular membranes undergo morphological changes for degradation and turnover of cytoplasmic organelles (27).

Imanitib mesylate and carvedilol combination treatment increases cell cycle arrest at G0/G1 phase similarly to imatinib mesylate for $72 \mathrm{~h}$. Cell cycle analysis showed that IM and the combination group promoted cell cycle arrest at G0/G1 phase concurrent with a significant reduction of the 

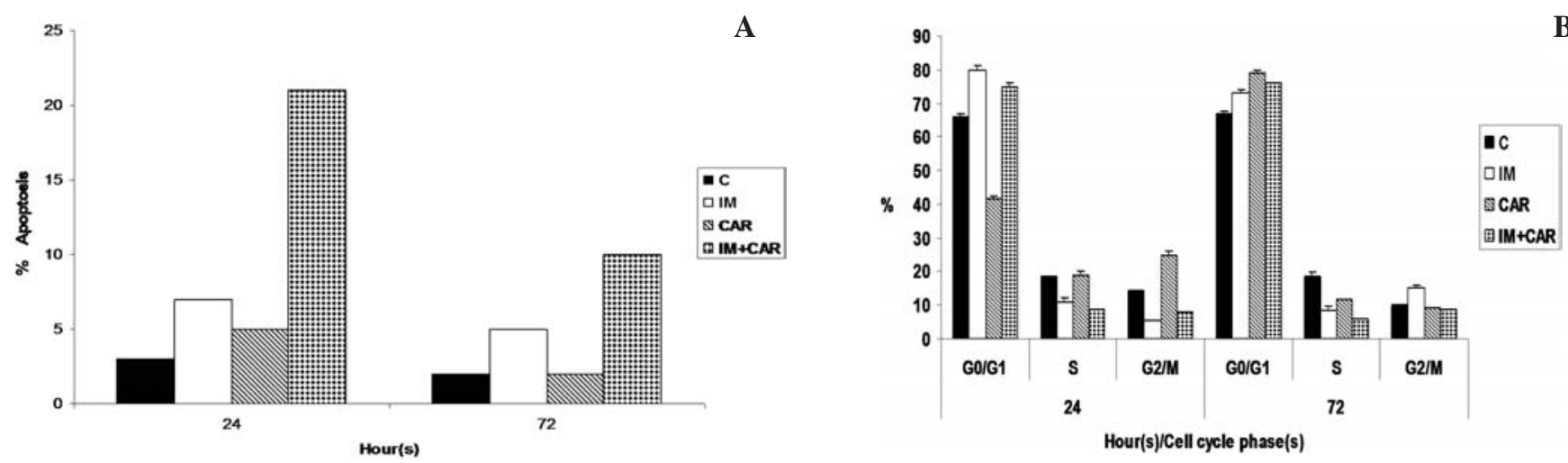

Figure 5. Flow cytometric analysis of alterations in apoptotic cell rates and cell cycle distributions of monolayer cultured C6 glioma cells. (A) The graph of apoptosis analysis. (B) The graph of cell cycle analysis. Cells were treated with drugs for a total period of $72 \mathrm{~h}$. Cells were harvested at the 24th and 72nd $\mathrm{h}$. The percentage of apoptotic cells and cells at G0/G1-, S- and G2/M-phases are plotted (\%). C, control; IM, imatinib mesylate; CAR, carvedilol; IM+CAR, imatinib mesylate with carvedilol.
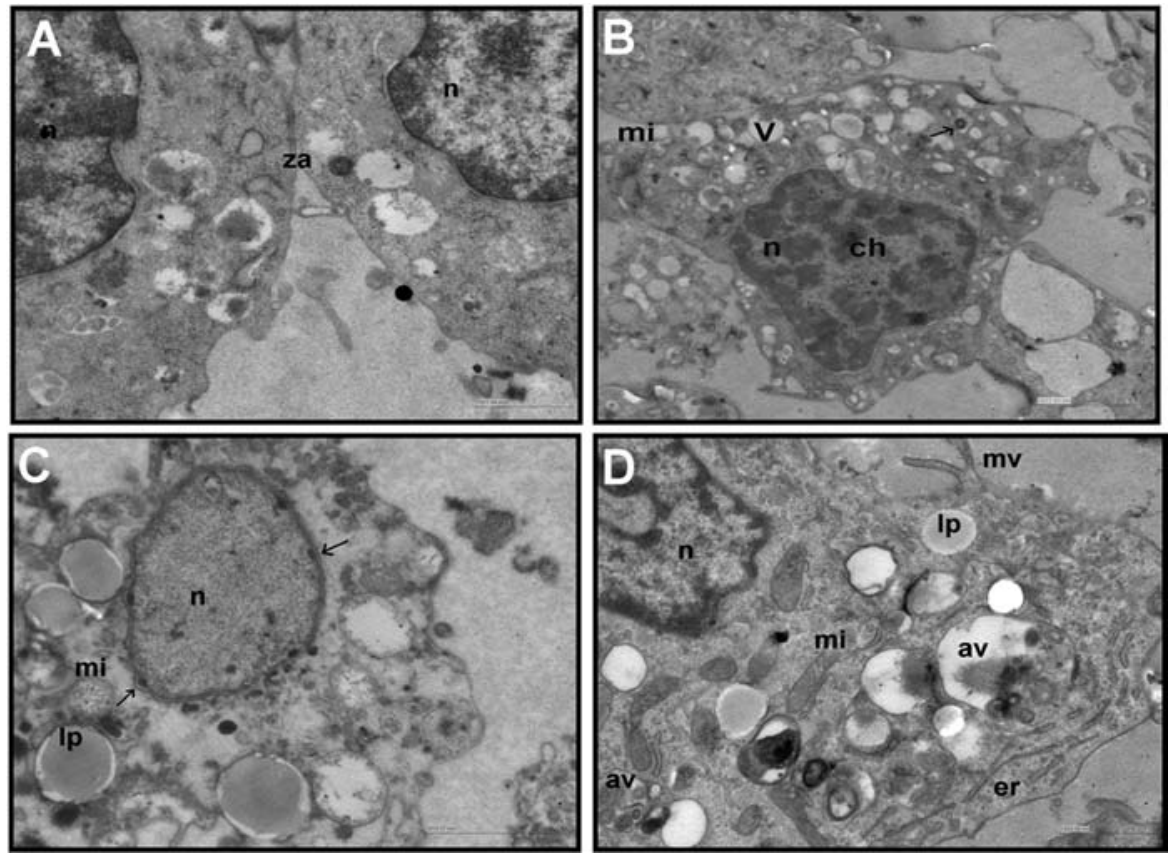

Figure 6. Transmission electron microscopic views of C6 glioma spheroids at the 72th $\mathrm{h}$. (A) The control group (original magnification, x20,000). (B) The imatinib mesylate group (original magnification, x10,000). (C) The carvedilol group (original magnification, x15,000). Arrow $(\rightarrow$ ) indicates that chromatin particules forced nucleus membrane to move cytoplasm. (D) The combination group (original magnification of x20,000). n, nucleus; za, zonula adherens; ch, chromatin; mi, mitochondria; v, vacuole; lp, lipid droplets; av, autophagic vacuoles; er, endoplasmic reticulum.

percentage of cells in $\mathrm{S}$ and $\mathrm{G} 2 / \mathrm{M}$ phases at $24 \mathrm{~h}$ (IM, $\mathrm{p}<0.0001$; the combi-nation group, $\mathrm{p}<0.01$ ) and $72 \mathrm{~h}$ (IM, $\mathrm{p}<0.01$; the combination group, $\mathrm{p}<0.001)$. In comparison with the combination group, IM induced a significant mild increase in the percentage of cells by $\sim 5 \%$ at $24 \mathrm{~h}(\mathrm{p}<0.01)$ and its effect in this proportion were similar at $72 \mathrm{~h}$. CAR arrested cells at G0/G1 phase in a time-dependent manner. Surprisingly, CAR induced a significant increase in the percentage of cells in $\mathrm{S}$ and $\mathrm{G} 2 / \mathrm{M}$ concurrent with a reduction of the percentage of cells in $\mathrm{G} 0 / \mathrm{G} 1$ at $24 \mathrm{~h}$ $(\mathrm{p}<0.00001)$, however CAR promoted cell cycle arrest at G0/G1 phase similar to IM but a significant increase in the proportion of cells by $\sim 3 \%$ compared to the combination at $72 \mathrm{~h}(\mathrm{p}<0.01)$ (Fig. 5B).
Assessment of spheroid morphology by TEM. After $72 \mathrm{~h}$ of culture, the untreated control group exhibited normal cellular morphology, but intracytoplasmic vacuoles were also noted (Fig. 6A). The IM-treated group exhibited flattening of the cell surface and severe plasma membrane blebbing; however, the plasma membrane remained intact. The nuclei showed apoptosis-like condensation and fragmentation. This treatment group also exhibited severe mitochondrial damage, foamyvacuolated cytoplasm, autophagic vacuoles and lytic changes (Fig. 6B). CAR treatment induced two types of changes; in some cells, increased numbers of mitochondria with severe cristae damage and/or blebbing of the cytoplasmic membrane were noted with a lack of pronounced damage to the nucleus. Disruption of cristae by IM was more severe than by CAR, 

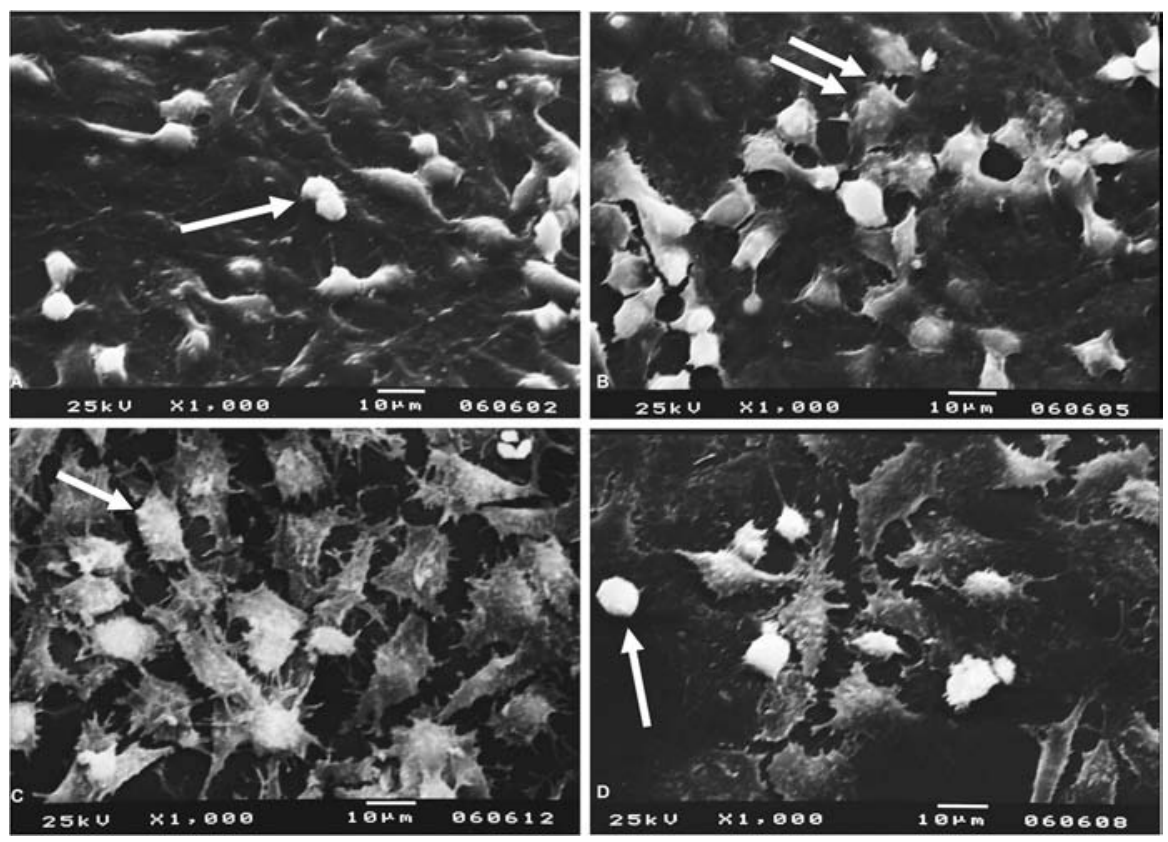

Figure 7. Scanning electron microscopic views of C6 glioma cells at the 72th h. (A) The control group (original magnification, x500). Arrow ( $\rightarrow$ ) indicates mitosis phase of cell. (B) The imatinib mesylate group (original magnification, x1000). Two arrows indicate blebbing of membrane. (C) The carvedilol group. Arrow represents cells which possess extended and angular body (original magnification, x750). (D) The combination treatment group. Long arrow indicates one of the cell type which was like a ball (original magnification, x750).

whereas in other cells, loss of nucleus accompanied by the same mitochondrial changes were seen (data not shown). Observed chromatin packaging in the CAR-treated group differentiated the group from the IM-treated group. Electron dense masses of chromatin granules were located mainly in the peripheral region of the nucleoplasm, and interestingly displaced the nuclear membrane to the cytoplasm, causing nuclear blebbing. An increase in vacuoles and huge lipid droplets were the most common changes. Lytic changes were also seen in cytoplasm. Some cells appeared to linger between apoptosis and necrosis (Fig. 6C). The IM and CAR combination group exhibited apoptosis morphology, mitochondria damage, and an increase in vacuoles, lipid droplets and autophagic vacuole-like structures were also observed (Fig. 6D). These findings suggest that combination treatment with IM and CAR resulted in enhanced morphological changes consistent with autophagy in C6 glioma cells as early as $24 \mathrm{~h}$ of treatment and that these changes were enhanced with a longer treatment period.

Assessment cell morphology by SEM. Following $72 \mathrm{~h}$ of culture, morphologic changes which were first seen after $24 \mathrm{~h}$ of treatment, were further enhanced. Untreated control C6 glioma cells were observed to have a spindle-shaped appearance and to frequently be in the active mitotic division phase (Fig. 7A). In the IM-treated group, cells possessed an angular morphology or globular appearance. IM treatment led to a sharp decrease in the number of microvilli and cell surface knobs (Fig. 7B). In the CAR-treated group, many cells developed a wide body and many short extensions on the cell surface (Fig. 7C). The combination-treatment group showed two different types of changes; some cells had a wide body and many short extensions on the cell surface, whereas other cells were not found to have any microvilli or extensions on the cell surface (Fig. 7D).

\section{Discussion}

Treatment of glioblastoma multiforme (GBM) with an effective chemotherapeutic regimen remains a challenge. It has been reported that IM inhibits the growth of glioma cells in preclinical models, but has poor distribution in the nervous system due to the fact that $\mathrm{p}-\mathrm{Gp}$ limits its efficacy in patients (14). The results of this study show that CAR provides a synergistic effect on IM-induced cytotoxicity in monolayer and spheroid cultures of malignant C6 glioma cells. Using a number of different techniques, we demonstrated that exposure of C6 glioma cells and spheroids to IM in combination with CAR leads to a reduction in 1) cell number, 2) spheroid volume, 3 ) the percentage of cells in S phase, 4) cAMP levels and 5) increases apoptosis.

Surprisingly, we found that CAR causes severe mitochondrial damage, as shown by TEM images of C6 glioma spheroids treated with CAR. Alterations of spheroid morphology revealed that CAR treatment induced mitochondrial damage, such as mitochondrial swelling, crista damage and formation of myelin figures inside the mitochondria. Contrary to our results, the study by Santos et al demonstrated CAR's cardioprotective effect through mitochondrial conservation when co-administrated with the cardiac toxin, doxorubicin (27). The group showed that CAR reduced cellular vacuolization in cardiac myocytes and prevented doxorubicin's inhibitory effect on mitochondrial respiration in both heart and liver cells. There are many reports on the protective effect of CAR on mitochondria via its antioxidant property (27-30). Similar to doxorubicin, IM's inhibitory 
effect on mitochondria has been proven with several findings, including those from the cardiotoxicity and cancer studies identifying mitochondria as a chief target of IM $(31,32)$. The starting point for this study was the potential survival inhibitory effect of CAR on a few malignant cell lines such as myelogenous leukemia K562, cervix carcinoma HeLa, melanoma Fem-X, breast tumour and osteosarcoma MG63 in vitro, shown by a previous study by Stanojkovic et al (20). In light of this study, CAR's inhibitory effect on C6 rat glioma cell line was an anticipated result, but if we consider the study of Santos and coworkers (27), prevention of IMinduced mitochondrial damage by carvedilol could have been one of the expected results. However, we find CAR in combination with IM augments IM-induced mitochondrial damage.

The alteration in cAMP levels of CAR-treated cells supports the morphologic findings of CAR-induced mitochondrial damage. C6 glioma cells possess native B-ADR and functional PDGFRs and thus proliferate in response to PDGF treatment. Stimulation of PDGFR leads to an increase in cAMP level $(33,34)$. G-protein coupled receptors, $\alpha$ and $\beta$-ADR, growth factor receptors, etc., are responsible for intracellular cAMP accumulation and changes in cAMP levels affect the selective activation of cAMP-dependent protein kinase-A isoforms. The chief source of cAMP is ATP, which is generated by mitochondria $(35,36)$. In our study, effective inhibition of cAMP levels was observed in the IM-treated group at $72 \mathrm{~h}$ of treatment and in both the CAR-treated group and the combination drug-treated group. Modulation of cAMP levels could also be interpreted as a CAR inhibitory effect on growth factor receptors such as PDGFR and B-ADR.

The effect of CAR treatment on cell number, BrDU-LI , cell cycle distribution and cellular morphology were other interesting points of our study. Although the apparent mean terminal elimination half-life of CAR generally ranges from 7 to $10 \mathrm{~h}$ (41) CAR's inhibitory effect on cell growth peaked after $48 \mathrm{~h}$ of treatment. Moreover, IM and CAR were similarly effective at reducing cell proliferation up to $72 \mathrm{~h}$ of treatment, at which point, CAR treatment reduced the cell count. In contrast to CAR, IM with a terminal half-life, ranging from 18 to $40 \mathrm{~h}$ (42), reduced BrDU uptake more effectively than CAR at $48 \mathrm{~h}$ of treatment and subsequently mildly lost its inhibitory effect, resulting in the same inhibitory results as CAR at $72 \mathrm{~h}$ of treatment. Results from flow cytometric cell cycle analysis were similar to CAR arrested cells at G0/G1 phase showing an effect in a time-dependent manner especially late in the incubation time. CAR induced a significant increase in the percentage of cells in $\mathrm{S}$ and G2/M at $24 \mathrm{~h}$, but CAR promoted cell cycle arrest at G0/G1 phase similar to IM but with a significant increase in the proportion of cells, which was different from the combination at $72 \mathrm{~h}$. In addition, surprisingly when singly applied CAR increased the percentage of cells in $\mathrm{S}$ and $\mathrm{G} 2 / \mathrm{M}$, the combination group efficiently increased the percentage of cells in G0/M at $24 \mathrm{~h}$. Others have shown a reduction of cells in $\mathrm{S}$ and $\mathrm{G} 2 / \mathrm{M}$ phase using monolayer cultured rat aortic smooth muscle cells treated and pulmonary artery smooth muscle cells with the same concentration of CAR $(43,44)$. Modulation of the cell cycle could also be interpreted as a CAR inhibitory effect on growth factor receptors such as PDGFR and $\beta$-ADR. Our SEM studies revealed that after $24 \mathrm{~h}$ of treatment, the morphology of cells in the CAR-treated group seemed similar to that of the control group, but following $72 \mathrm{~h}$, more cells developed a wide body with short cytoplasmic extentions, suggesting increased sensitivity to CAR at $72 \mathrm{~h}$ of treatment.

Alterations in spheroid morphology revealed that IM induced and increased the cellular autophagy response in our study. Three different studies regarding the IM effect on autophagy were done by Ertmer et al and Carew et al (4547). Ertmer et al described, for the first time, the process of autophagy as the mode of action of IM (45). They considered that autophagy may promote apoptosis in cancer cells. They revealed that the IM dose-dependently activates the cellular autophagy response in mammalian cells, independent of tissue type, species origin, or immortalization status of cells. These findings are in contrast to Ertmer et al (45), Carew et al $(46,47)$ who considered that autophagy may promote cancer cell survival and play an important role in chemoresistance of cancer to some therapeutic agents, which typically induce an apoptotic response. They showed that disrupting autophagy augmented the anticancer activity of histone deacetylase (HDAC) inhibitor suberoylanilide hydroxamic acid (SAHA) in IM-refractory chronic myelogenous leukemia (CML) and in IM-resistant primary CML cells from patients bearing mutations in Bcr-Abl, including the T315I mutation that causes resistance to currently utilized tyrosine kinase inhibitors and translates into a very poor clinical prognosis.

The observed reduction of cellular proliferation and downregulation of metabolic activity by IM and CAR combination treatment led us to ask if such combination treatment also induces apoptosis in C6 glioma cells. To answer the question, we performed Annexin-V-FITC/PI staining to assess apoptosis in cells treated either individually with IM or CAR or in combination. In the present study, especially in light of the electron micrograph findings with Annexin-V-FITC and PI double-staining results together, when IM was used in combination, the induction of autophagy by IM might represent an additional mechanism to induce apoptosis in C6 glioma cells, like the study of Ertmer et al (45). Besides, if the mode of action of CAR was evaluated, although there were no reports on the connection of carvedilol and autophagy, the induction and/or increase of autophagy by CAR can be interpreted as an additional mechanism of CAR to induce growth arrest and promote apoptosis in C6 glioma cells.

Spheroids, known as an in vivo-like tissue culture model, are the best adapted model for maintainance of in vitro resistance properties of cells (48). In this study, all drug treatments reduced spheroid volume and BrDU-LI. The most effective reduction of spheroid volume was observed in the CAR-treated group. Interestingly although CAR induced a decrease in spheroid volume, similar to the BrDU-LI results of monolayer cultures, CAR only mildly inhibited BrDU-LI uptake of spheroids. This result suggests that although CAR does affect cell growth, it does not play a large role in $\mathrm{S}$ phase inhibition. Taken together, these results suggest that carvedilol showed antitumor effects at glioma treatment through mitochondria damage and carvedilol enhanced the effects of imatinib mesylate. 
Although CAR is not, strictly speaking, a chemotherapeutic agent, according to our study, CAR may be an additional compound needed in treatment of glioma. Our results also should be directed to animal models in order to confirm the efficacy and safety of carvedilol and the combination treatments.

\section{Acknowledgements}

The present work was supported by the Research Fund of Istanbul University, project number 484/05052006. Imatinib was provided by Novartis AG, Basel, Switzerland and Novartis AG, Istanbul, Turkey. The authors thank Fusun Oncu and Ebru Karabulut for their technical assistance. Some parts of this manuscript was presented at the12th World Congress on Advances in Oncology and and 10th International Symposium on Molecular Medicine, October, 2007, Hersonissos, Crete, Greece.

\section{References}

1. Arora A and Scholar EM: Role of tyrosine kinase inhibitors in cancer therapy. J Pharmacol Exp Ther 315: 971-979, 2005.

2. Heinrich MC, Blanke CD, Druker BJ, et al: Inhibition of KIT tyrosine kinase activity: a novel molecular approach to the treatment of KIT-positive malignancies. J Clin Oncol 20: $1692-1703,2002$

3. Buchdunger E, Cioffi CL, Law N, et al: Abl protein-tyrosine kinase inhibitor STI571 inhibits in vitro signal transduction mediated by c-kit and platelet-derived growth factor receptors. J Pharmacol Exp Ther 295: 139-145, 2000.

4. Yu C, Krystal G, Dent P, et al: Flavopiridol potentiates STI571induced mitochondrial damage and apoptosis in Bcr-Ablpositive human leukemia cells. Clin Cancer Res 8: 2976-2984, 2002.

5. Uziel O, Fenig E, Nordenberg J, et al: Imatinib mesylate (Gleevec) downregulates telomerase activity and inhibits proliferation in telomerase-expressing cell lines. Br J Cancer 92: 1881-1891, 2005.

6. Deininger M, Buchdunger E and Druker BJ. The development of imatinib as a therapeutic agent for chronic myeloid leukemia. Blood 105: 2640-2653, 2005.

7. Tarn C, Skorobogatko YV, Taguchi T, et al: Therapeutic effect of imatinib in gastrointestinal stromal tumors: AKT signalingdependent and -independent mechanisms. Cancer Res 66: 5477-5486, 2006.

8. Kilic T, Alberta JA, Zdunek PR, et al: Intracranial inhibition of platelet-derived growth factor-mediated glioblastoma cell growth by an orally active kinase inhibitor of the 2-phenylaminopyrimidine class. Cancer Res 60: 5143-5150, 2000.

9. Krystal GW, Honsawek S, Litz J, et al: The selective tyrosine kinase inhibitor STI571 inhibits small cell lung cancer growth. Clin Cancer Res 6: 3319-3326, 2000

10. Mitsiades CS, Sykoutri D, McMullan C, et al: Effect of imatinib mesylate (Gleevec) on anaplastic thyroid carcinoma cell lines. J Clin Endocrinol Metab 88: 5043-5044, 2003.

11. Kim SJ, Uehara H, Yazici S, et al: Targeting platelet-derived growth factor receptor on endothelial cells of multidrugresistant prostate cancer. J Natl Cancer Inst 98: 783-793, 2006.

12. O'Reilly T, Wartmann M and Maira SM: Patupilone (epothilone B, EPO906) and imatinib (STI571, Glivec) in combination display enhanced antitumour activity in vivo against experimental rat C6 glioma. Cancer Chemother Pharmacol 55: 307-317, 2005.

13. Lokker NA, Sullivan CM, Hollenbach SJ, et al: Platelet-derived growth factor (PDGF) autocrine signaling regulates survival and mitogenic pathways in glioblastoma cells: evidence that the novel PDGF-C and PDGF-D ligands may play a role in the development of brain tumors. Cancer Res 62: 3729-3735, 2002.

14. Dai H, Marbach P, Lemaire M, et al: Distribution of STI-571 to the brain is limited by P-glycoprotein-mediated efflux. J Pharmacol Exp Ther 304: 1085-1092, 2003.
15. Breedveld P, Beijnen JH and Schellens JH: Use of P-glycoprotein and BCRP inhibitors to improve oral bioavailability and CNS penetration of anticancer drugs. Trends Pharmacol Sci 27: 17-24, 2006.

16. Mochizuki M, Yano M, Oda T, et al: Scavenging free radicals by low-dose carvedilol prevents redox-dependent $\mathrm{Ca}^{2+}$ leak via stabilization of ryanodine receptor in heart failure. J Am Coll Cardiol 49: 1722-1732, 2007.

17. Coats A: Use of carvedilol in the treatment of heart failure. Hosp Med 64: 288-291, 2003.

18. Book WM: Carvedilol: a nonselective beta blocking agent with antioxidant properties. Congest Heart Fail 8: 173-177. Erratum in: Congest Heart Fail 8: 240, 2002.

19. Stanojkovic TP, Zizak Z and Mihailovic-Stanojevic N: Inhibition of proliferation on some neoplastic cell lines-act of carvedilol and captopril. J Exp Clin Cancer Res 24: 387-395, 2005.

20. Liu CP and Jan CR: Effect of carvedilol on $\mathrm{Ca}^{2+}$ movement and cytotoxicity in human MG63 osteosarcoma cells. Basic Clin Pharmacol Toxicol 95: 59-65, 2004.

21. Bachmakov I, Werner U, Endress B, et al: Characterization of beta-adrenoceptor antagonists as substrates and inhibitors of the drug transporter P-glycoprotein. Fundam Clin Pharmacol 20: 273-282, 2006.

22. Moe G: Carvedilol in the treatment of chronic heart failure. Expert Opin Pharmacother 2: 831-843, 2001.

23. Santini MT and Rainaldi G: Three-dimensional spheroid model in tumor biology. Pathobiology 67: 148-157, 1999.

24. Altinoz MA, Bilir A, Del Maestro RF, et al: Noscapine and diltiazem augment taxol and radiation-induced S-phase arrest and clonogenic death of C6 glioma in vitro. Surg Neurol 65: 478-485, 2006.

25. Conger AD and Ziskin MC: Growth of mammalian multicellular tumor spheroids. Cancer Res 43: 556-560,1983.

26. Kaygisiz Z, Erkasap N, Yazihan N, et al: Erythropoietin changes contractility, cAMP, and nitrite levels of isolated rat hearts. J Physiol Sci 56: 247-251, 2006.

27. Santos DL, Moreno AJ, Leino RL, et al: Carvedilol protects against doxorubicin-induced mitochondrial cardiomyopathy. Toxicol Appl Pharmacol 185: 218-227, 2002.

28. Oliveira PJ, Bjork JA, Santos MS, et al: Carvedilol-mediated antioxidant protection against doxorubicin-induced cardiac mitochondrial toxicity.Toxicol Appl Pharmacol 200: 159-168, 2004.

29. Spallarossa P, Garibaldi S, Altieri P, et al: Carvedilol prevents doxorubicin-induced free radical release and apoptosis in cardiomyocytes in vitro. J Mol Cell Cardiol 37: 837-846, 2004.

30. Oliveira PJ, Goncalves L, Monteiro P, et al: Are the antioxidant properties of carvedilol important for the protection of cardiac mitochondria? Curr Vasc Pharmacol 3: 147-158, 2005.

31. Kerkela R, Grazette L, Yacobi R, et al: Cardiotoxicity of the cancer therapeutic agent imatinib mesylate. Nat Med 12: 908-916, 2006.

32. Jacquel A, Herrant M, Legros L, et al: Imatinib induces mitochondria-dependent apoptosis of the Bcr-Abl-positive K562 cell line and its differentiation toward the erythroid lineage. FASEB J 17: 2160-2162. Erratum in: FASEB J 17: 2347, 2003.

33. Sokolowska P and Nowak JZ: Constitutive activity of betaadrenergic receptors in C6 glioma cells. Pharmacol Rep 57: 659-663, 2005.

34. Grobben B, De Deyn PP and Slegers H: Rat C6 glioma as experimental model system for the study of glioblastoma growth and invasion. Cell Tissue Res 310: 257, 2002.

35. Jaiswal BS and Conti M: Calcium regulation of the soluble adenylyl cyclase expressed in mammalian spermatozoa. Proc Natl Acad Sci USA 100: 10676-10681, 2003.

36. Baillie GS and Houslay MD: Arrestin times for compartmentalised cAMP signalling and phosphodiesterase- 4 enzymes. Curr Opin Cell Biol 17: 129-134, 2005.

37. Hyde SC, Emsley P, Hartshorn MJ, et al: Structural model of ATP-binding proteins associated with cystic fibrosis, multidrug resistance and bacterial transport. Nature 346: 362-365, 1990.

38. Shapiro AB and Ling V: Reconstitution of drug transport by purified P-glycoprotein. J Biol Chem 270: 16167-16175, 1995.

39. Sharom FJ, Yu X and Doige CA: Functional reconstitution of drug transport and ATPase activity in proteoliposomes containing partially purified P-glycoprotein. J Biol Chem 268: 24197-24202, 1993.

40. Sharom FJ, Yu X, DiDiodato G, et al: Synthetic hydrophobic peptides are substrates for P-glycoprotein and stimulate drug transport. Biochem J 320: 421-428, 1996. 
41. Vanderhoff BT, Ruppel HM and Amsterdam PB: Carvedilol: the new role of beta blockers in congestive heart failure. Am Fam Physician 58: 1627-1642, 1998.

42. Bornhäuser M, Pursche S, Bonin M, et al: Elimination of Imatinib mesylate and its metabolite N-desmethyl-Imatinib. J Clin Oncol 23: 3855-3856, 2005.

43. Sung CP, Arleth AJ, Eichman C, et al: Carvedilol, a multipleaction neurohumoral antagonist, inhibits mitogen-activated protein kinase and cell cycle progression in vascular smooth muscle cells. J Pharmacol Exp Ther 283: 910-917, 1997.

44. Fujio H, Nakamura K, Matsubara H, et al: Carvedilol inhibits proliferation of cultured pulmonary artery smooth muscle cells of patients with idiopathic pulmonary arterial hypertension. J Cardiovasc Pharmacol 47: 250-255. Erratum in: J Cardiovasc Pharmacol 47: table of contents, 2006.
45. Ertmer A, Huber V, Gilch S, et al: The anticancer drug imatinib induces cellular autophagy. Leukemia 21: 936-942, 2007.

46. Carew JS, Nawrocki ST and Cleveland JL: Modulating autophagy for therapeutic benefit. Autophagy 3: 464-467, 2007.

47. Carew JS, Nawrocki ST, Kahue CN, et al: Targeting autophagy augments the anticancer activity of the histone deacetylase inhibitor SAHA to overcome Bcr-Abl-mediated drug resistance. Blood 110: 313-322, 2007.

48. Takagi A, Watanabe M, Ishii Y, et al: Three-dimensional cellular spheroid formation provides human prostate tumor cells with tissue-like features. Anticancer Res 27: 45-53, 2007. 